\title{
Inhibitory Effects of Euphorbia ebracteolata Hayata Extract ECB on Melanoma-Induced Hyperplasia of Blood Vessels in Zebrafish Embryos
}

\author{
Wenjing Dong $\mathbb{D}^{1},{ }^{1}$ Xinyue Han $\left(D,{ }^{1}\right.$ Chao Bao, ${ }^{1}$ Saijilahu Tai, ${ }^{2}$ Yuxia Bai $\left(D,{ }^{3}\right.$ Liang Xu, ${ }^{4}$ \\ Jingfeng Yang, ${ }^{1}$ TinChung Leung $\left(\mathbb{D},{ }^{5}\right.$ Wuliji Ao $\mathbb{D}^{3},{ }^{3}$ and Wu Dong $\left(\mathbb{1}{ }^{1}\right.$ \\ ${ }^{1}$ Inner Mongolia Key Laboratory of Toxicant Monitoring and Toxicant and Toxicology, College of Animal Science and Technology, \\ Inner Mongolia University for Nationalities, Tongliao, Inner Mongolia 028000, China \\ ${ }^{2}$ Mongolian State University of Education, Ulaanbaatar 210648, Mongolia \\ ${ }^{3}$ Inner Mongolia Research Institute of Traditional Mongolian Medicine Engineering Technology/College of Mongolian \\ Medicine and Pharmacy, Inner Mongolia University for Nationalities, Tongliao 028000, China \\ ${ }^{4}$ Inner Mongolia Key Laboratory for the Natural Products Chemistry and Functional Molecular Synthesis, \\ College of Chemistry and Chemical Engineering, Inner Mongolia University for Nationalities, Tongliao, \\ Inner Mongolia 028000, China \\ ${ }_{5}^{5}$ Julius L. Chambers Biomedical Biotechnology Research Institute, Dept of Biological \& Biomedical Sciences, \\ North Carolina Central University, Kannapolis, NC 28081, USA
}

Correspondence should be addressed to TinChung Leung; tleung@nccu.edu, Wuliji Ao; wuliji@126.com, and Wu Dong; dongwu2002@hotmail.com

Received 24 January 2021; Revised 25 March 2021; Accepted 5 April 2021; Published 27 April 2021

Academic Editor: Jie Liu

Copyright ( $\odot 2021$ Wenjing Dong et al. This is an open access article distributed under the Creative Commons Attribution License, which permits unrestricted use, distribution, and reproduction in any medium, provided the original work is properly cited.

Melanoma is a serious malignant form of skin cancer. Euphorbiaceae compound B (ECB, 2,4-dihydroxy-6-methoxy-3-methylacetophenone) is an acetophenone compound that is isolated from Euphorbia ebracteolata Hayata (EEH), an herbaceous perennial, and has antitumor activity. Here, we transplanted human melanoma cells into zebrafish embryos to establish a zebrafish/melanoma model. We showed that this model can be used to evaluate the therapeutic effect of EEH and ECB and discussed its potential mechanism of action. The results showed that ECB was an active ingredient of EEH in inhibiting melanoma-induced hyperplasia of blood vessels in zebrafish embryos, similar to the angiogenic inhibitor vatalanib. ECB inhibited the number and length of subintestinal veins $(p<0.05)$, as well as the distribution of melanoma in zebrafish embryos $(p<0.05)$. More importantly, unlike vatalanib, ECB only inhibited melanoma-induced abnormal and excessive growth of blood vessels in xenografts. In addition, ECB inhibited the mRNA expression of vegfr2 and vegfr3 in zebrafish. Both vegfr 2 and vegfr 3 are essential genes that regulate blood vessel formation and upregulate the expression of $p 53$ and casp $3 a$ genes in zebrafish. Together, the above-mentioned results indicate that ECB has a potential antimelanoma effect in vivo, which may be mediated by inhibiting vascular endothelial growth factor receptors.

\section{Introduction}

Malignant melanoma is the most serious type of skin cancer, which is caused by hyperplasia of melanocytes in the skin. In the body, melanoma cells can rapidly metastasize, and patients with metastatic melanoma have a survival of no more than five years [1]. Euphorbiaceae compound B (ECB, 2, 4- dihydroxy-6-methylbenzoidone) is a compound of benzene ketones in the Euphorbia ebracteolata Hayata (EEH) and Euphorbia fischeriana Steud [2, 3]. Studies that focused on the anticancer effects of diterpenoids in Euphorbiaceae have been reported; however, studies on ECB are limited. Blocking the pathway underlying tumor angiogenesis may be a unique way to block the growth of blood vessels that 
support tumor growth [4]. The zebrafish model can be used as a viable model for whole-animal screening using small molecules that affect tumor angiogenesis [5]. In particular, the subintestinal vein (SIV) of zebrafish/tumor xenografts can be visualized noninvasively in real time using vascularspecific transgenic zebrafish or can be stained using blood vessel dye and visualized under a microscope [6]. Koenig et al. showed that vegfaa was expressed at the site of intestinal vascularization and might provide a guiding signal and that vegfc overexpression could lead to SIV overgrowth. The authors suggested that (1) Vegfaa signaling might regulate endothelial cells to migrate out of the existing vasculature and subsequently merge to form intestinal blood vessels and that (2) Vegfc could induce growth of the SIV [7]. PTK787, a potent angiogenic inhibitor, inhibits vascular endothelial growth factor receptors (VEGFR) and plateletderived growth factor receptor (PDGFR) tyrosine kinase and blocks cell proliferation and cell survival, resulting in cell death and reduced blood vessel density in leiomyosarcoma cells, as well as in zebrafish xenografts $[8,9]$. The potent B-raf inhibitor PLX4720 (PLX) that acts against melanoma reduced the binding of C-MYC to the VEGF promoter to reduce the expression of VEGF in melanoma cells and increased the infiltration of adoptively transferred $\mathrm{T}$ cells, thereby leading to an antitumor effect [10].

As a model for high-throughput screening of anticancer drugs, zebrafish have been shown to be a potential model. In this study, we used $\operatorname{Tg}(f l k 1: G F P)$ transgenic zebrafish as a model to quantify fluorescent blood vessels after transplantation of melanoma cells into zebrafish embryos. We aimed to use this model for screening the antimelanoma effect of ECB through tumor-induced angiogenesis and metastasis. The potential underlying mechanisms involved were also studied (see Graphic abstract).

\section{Materials and Methods}

2.1. Chemicals and Reagents. Human melanoma cells (A2058 cells), originated from a 43-year-old Caucasian adult male, were obtained from the American Type Culture Collection (ATCC, Manassas, VA, USA). Human umbilical vein endothelial cells (HUVECs) were extracted from human umbilical cord vein tissue and obtained from ScienCell (San Diego, CA, USA). The Vybrant ${ }^{\mathrm{TM}}$ CM-DiI cell-labeling solution (CM-DiI) was purchased from Invitrogen (Carlsbad, CA, USA). Vatalanib (PTK) and PLX-4720 (PLX) were purchased from MedChemExpress (Monmouth Junction, NJ, USA). ECB was purchased from the National Institutes for Food and Drug Control (Beijing, China). Other compounds and reagents were purchased from Sigma-Aldrich (St. Louis, MO, USA).

\subsection{Preparation and High-Performance Liquid Chromatog-} raphy Analysis of EEH. Euphorbia ebracteolata Hayata (EEH) was provided by Liang Xu's Lab, and EEH was authenticated and analyzed by $\mathrm{Dr}$. Liang $\mathrm{Xu}$ from the Inner Mongolia Key Laboratory for the Natural Products Chemistry and Functional Molecular Synthesis, College of
Chemistry and Chemical Engineering, Inner Mongolia University for Nationalities (Tongliao, China). A total of $10 \mathrm{~g}$ EEH powder was soaked into $100 \mathrm{~mL}$ of $90 \%$ ethanol at room temperature for 24 hours. The supernatant was concentrated to $10 \mathrm{~mL}$ after the mixture was refluxed for 3 hours, filtered through a $0.22 \mu \mathrm{m}$ filter, and used as a $1 \mathrm{~g} / \mathrm{mL}$ stock solution. ECB is a major ingredient of EEH (Figure S1) [11].

2.3. Zebrafish Breeding. $\operatorname{Tg}(f l k 1: G F P)$ zebrafish were donated by the Chinese Academy of Sciences and raised in a zebrafish breeding system (Environ Science, Beijing, China) at a water temperature of $28.5^{\circ} \mathrm{C}$ and a $14: 10$-hour day-night cycle. The zebrafish breeding and testing process was approved by the Animal Protection Association of Inner Mongolia University for Nationalities (Tong Liao, China).

\subsection{Establishment of a Zebrafish/Melanoma Xenograft Model.} Melanoma cells were labeled with a fluorescence dye (CMDiI), and zebrafish/melanoma xenografts were cultured in a $35^{\circ} \mathrm{C}$ incubator for $2 \mathrm{~h}$, then transferred to a $28.5^{\circ} \mathrm{C}$ incubator until 24 hours after injection (hpi) and 48 hpi. After $6 \mathrm{~h}$ of culture (6 hpi), zebrafish/melanoma xenografts that showed the same amount of melanoma cells under a fluorescence microscope were selected for further analysis.

2.5. EEH or ECB Treatment and Calculation of Blood Vessel Number, Length, and CM-DiI Labeling (Melanoma Cells) Area in Zebrafish. Zebrafish embryos were imaged using an inverted fluorescence microscope (Olympus IX73, Tokyo, Japan) at 24 hpi to capture SIV blood vessels and melanoma fluorescence in zebrafish embryos. ImageJ software $\mathrm{NIH}$, Bethesda, MD, USA) was used to measure the length of SIV ectopic blood vessels, the CM-DiI labeling area, and the number of ectopic blood vessels. After imaging at $24 \mathrm{hpi}, 10$ zebrafish xenografts were placed in a 6-well plate and treated with EEH, ECB, PTK, PLX, or buffer for the control group. Zebrafish xenografts were cultured for an additional $24 \mathrm{~h}$ prior to a second round of fluorescence imaging at $48 \mathrm{hpi}$ for quantification.

2.6. Cell Culture and Labeling. A2058 cells and/or HUVECs were cultured in a $37^{\circ} \mathrm{C}$ cell culture incubator at $5 \% \mathrm{CO}_{2}$. Tumor cells at a confluency of $60 \%-70 \%$ were labeled with $\mathrm{CM}$-DiI for $20 \mathrm{~min}$ at $37^{\circ} \mathrm{C}$, washed 3 times, 10 minutes with HBSS, and cultured overnight at $37^{\circ} \mathrm{C}$. The next day, cells were trypsinized and collected for microinjection.

2.7. Total RNA Isolation and Quantitative Real-Time Polymerase Chain Reaction. Total RNA was extracted using the TRIzol reagent [12]; vegfa, vegfr 2 , vegfr $3, p 53$, casp $3 a$, and $18 \mathrm{~s}$ (supplemental data: Table S1) genes were used for quantitative real-time PCR. As an internal reference, $18 \mathrm{~S}$ rRNA was used. The delta-delta $\mathrm{Ct}\left(2^{-\triangle \triangle \mathrm{CT}}\right)$ method was used to calculate the relative change in gene expression. 
2.8. Statistical Analysis. For statistical analysis, GraphPad Prism 5 software (GraphPad Software Inc., La Jolla, CA, USA) was used. Differences between groups were analyzed using one-way analysis of variance (ANOVA) followed by Tukey's post hoc test. Significance levels were set to ${ }^{*} p<0.05 ;{ }^{* *} p<0.01$; and ${ }^{* * *} p<0.001$.

\section{Results}

3.1. Inhibitory Effect of EEH or ECB on Melanoma-Induced Hyperplasia of Blood Vessels in Zebrafish Embryos. A2058 cells were microinjected beneath the surface of the yolk area at the $48 \mathrm{hpf}$ stage in transgenic $\operatorname{Tg}(f l k 1: G F P)$ zebrafish embryos. At $24 \mathrm{hpi}, 50 \mu \mathrm{g} / \mathrm{mL}$ of EEH was added to the embryo medium of the zebrafish/melanoma xenograft, containing the injected cells labeled with red fluorescent dye (CM-DiI, Invitrogen). Tumor-induced angiogenesis was quantified by evaluating the number and length of ectopic vessels in the SIV of zebrafish/tumor xenografts at 24 and $48 \mathrm{~h}$ after injection (24 and $48 \mathrm{hpi}$ ) (Figure 1). The length of the SIV ectopic blood vessels in control HUVECs and A2058 cells was measured. Our data showed that the length of SIV ectopic blood vessels in the A2058 group was significantly higher than that in the control group at both 24 and $48 \mathrm{hpi}(p<0.001)$. In addition, treatment of xenografts with $50 \mu \mathrm{g} / \mathrm{mL}$ EEH reduced the length of SIV ectopic blood vessels of A2058 xenografts. To verify the results on ectopic vessel length, we also quantified the number of ectopic vessels in each xenograft and found that EEH had a more clear effect on the inhibition of tumor angiogenesis in A2058 xenografts at 48 hpi $(p<0.05)$.

Similar to EEH experiments at $24 \mathrm{hpi}, 20 \mu \mathrm{g} / \mathrm{mL}$ of ECB and $1 \mu \mathrm{M}$ of PTK were added to the embryo medium of the zebrafish/melanoma xenograft, and tumor-induced angiogenesis was quantified by evaluating the number and length of ectopic vessels in the SIV of zebrafish/tumor xenografts at 24 and 48 hpi (Figure 2). The length of SIV ectopic blood vessels in control HUVECs and A2058 cells was measured. Our data showed that the length of SIV ectopic blood vessels in the A2058 group was significantly higher than that in the control group at both 24 and $48 \mathrm{hpi}(p<0.01)$. In addition, treatment of xenografts with $20 \mu \mathrm{g} / \mathrm{mL}$ ECB or $1 \mu \mathrm{M}$ PTK, which is antiangiogenic, significantly reduced the length of SIV ectopic blood vessels of A2058 xenografts $(p<0.001)$. Compared with control HUVECs, the length of ectopic vessels in both of ECB or PTK groups was not significantly different $(p>0.05)$ (Figure 2). Because the number of ectopic vessels was very small, it would be unable to compare the differences between treatments. To verify the results on ectopic vessel length, we also quantified the number of ectopic vessels in each xenograft and found that both ECB and PTK had a similar effect on the inhibition of tumor angiogenesis in A2058 xenografts at $48 \mathrm{hpi}(p<0.05)$. No significant differences were observed between A2058 cells and control HUVECs.

In embryos that were treated with different concentrations of ECB and PTK, no significant differences were observed at $72 \mathrm{hpf}$ in perimeters or the area of the SIV between ECB groups and the control group. However, the positive control group that was treated with $1 \mu \mathrm{M}$ PTK showed reduction in both area and perimeter (length) of SIV blood vessels of the SIV compared with the control group/or the $20 \mu \mathrm{g} / \mathrm{mL}$ ECB-treated group $(p<0.05)$. In addition, intersegment vessels (ISVs), as well as trunk artery and veins, were mostly missing in the $1 \mu \mathrm{M}$ PTKtreated group. Therefore, our findings suggested that ECB treatment did not affect normal angiogenesis in zebrafish embryos (Figure 3). This contrasted with the antiangiogenic molecule PTK that showed to be a potent inhibitor of all known VEGFRs (VEGFR-1, VEGFR-2, and VEGFR-3).

3.2. Effect of ECB on the Metastasis of Melanoma Cells in Zebrafish Xenografts. The same number of melanoma cells $\left(3 \times 10^{7}\right.$ cells $\left./ \mathrm{mL}\right)$ was injected into the superficial region of the yolk sac after the zebrafish developed for $48 \mathrm{~h}$. The metastatic effect of A2058 cells was observed in the zebrafish embryos at $6 \mathrm{hpi}, 24 \mathrm{hpi}$, and $48 \mathrm{hpi}$ (Figure 4). At $24 \mathrm{hpi}$, $20 \mu \mathrm{g} / \mathrm{mL}$ ECB and the melanoma inhibitor PLX were added and zebrafish/tumor xenografts were analyzed after $24 \mathrm{~h}$. The data showed that the area of CM-DiI labeling (melanoma cells) at $6 \mathrm{hpi}, 24 \mathrm{hpi}$, and $48 \mathrm{hpi}$ in zebrafish embryos was $0.009 \mathrm{~mm}^{2}, 0.012 \mathrm{~mm}^{2}$, and $0.013 \mathrm{~mm}^{2}$, respectively. In zebrafish, the area of A2058 cells was reduced to $0.005 \mathrm{~mm}^{2}$ after treatment with $20 \mu \mathrm{g} / \mathrm{mL}$ ECB, and the area of A2058 cells was reduced to $0.004 \mathrm{~mm}^{2}$ after treatment with $1 \mu \mathrm{M}$ PLX for $24 \mathrm{~h}(p<0.001)$ (Figure $4(\mathrm{~g}))$.

3.3. Effect of ECB on Blood Vessels and the Expression of Apoptosis-Associated Genes in a Zebrafish/Melanoma Xenograft Model. A2058 cells were labeled with CM-DiI, injected into zebrafish embryos at $48 \mathrm{hpf}$, and treated with 0 (Control) or $20 \mu \mathrm{g} / \mathrm{mL}$ ECB or $1 \mu \mathrm{M}$ PTK for $24 \mathrm{~h}$ from 72 hpf (24 hpi). Embryos were collected for the quantification of mRNA expression of vegfa, vegfr 2 , and vegfr 3 at $96 \mathrm{hpf}$ (48 hpi) (Figure 5). The data showed that ECB or the vascular inhibitor PTK downregulated the mRNA expression of vegfa. Furthermore, PTK significantly downregulated the mRNA expression of vegfr2, which was 0.6-fold compared with that of the control group. Compared with the control group, ECB or PTK significantly downregulated the mRNA expression of vegfr 3 by 0.5 -fold and 0.38 -fold, respectively $(p<0.05)$. Furthermore, compared with the A2058 cells-injected group, ECB significantly increased the mRNA expression of $p 53$ by 2.3 -fold and increased that of casp $3 a$ by 2.9 -fold $(p<0.05)$. No significant changes were observed for PLX groups $(p>0.05)$ (Figure 6).

\section{Discussion}

To evaluate the inhibitory effect of EEH and ECB on melanoma, $\operatorname{Tg}(f l k 1: G F P)$ transgenic zebrafish embryos were used as an in vivo model. In brief, A2058 cells were labeled with red fluorescent dye and microinjected into $48 \mathrm{hpf}$ zebrafish embryos. Therefore, the labeled cancer cells can be monitored for tumor growth and tumor-induced neovascularization in developmental zebrafish embryos. 


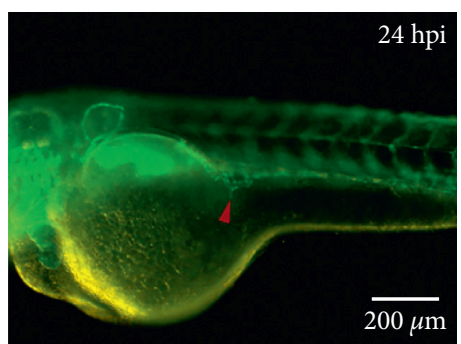

(a)

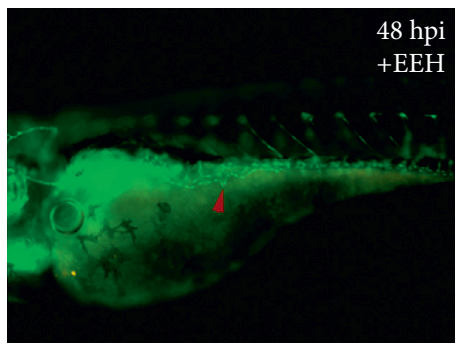

(c)

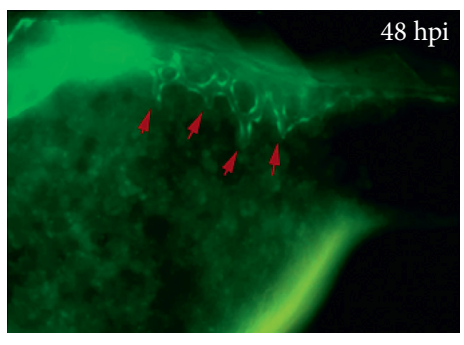

(e)

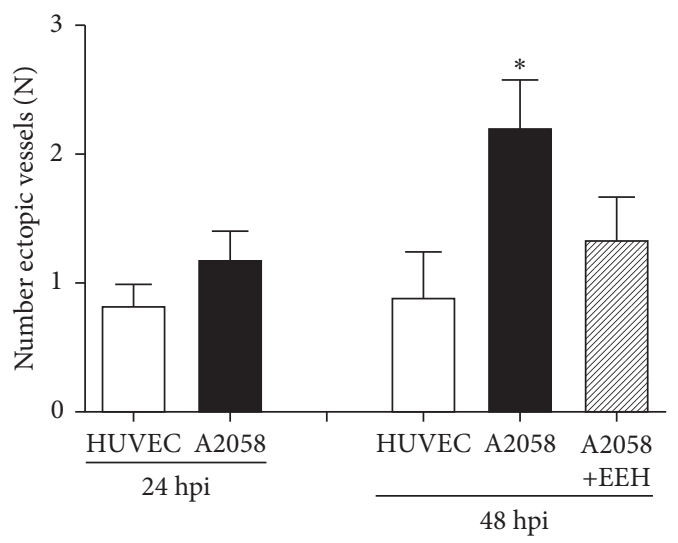

(g)

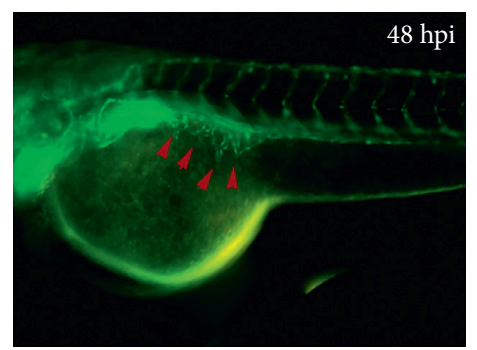

(b)

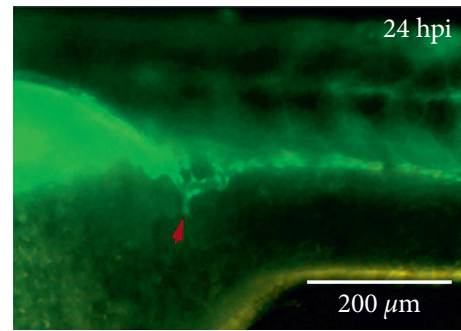

(d)

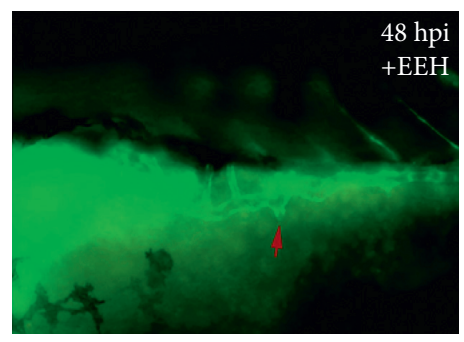

(f)

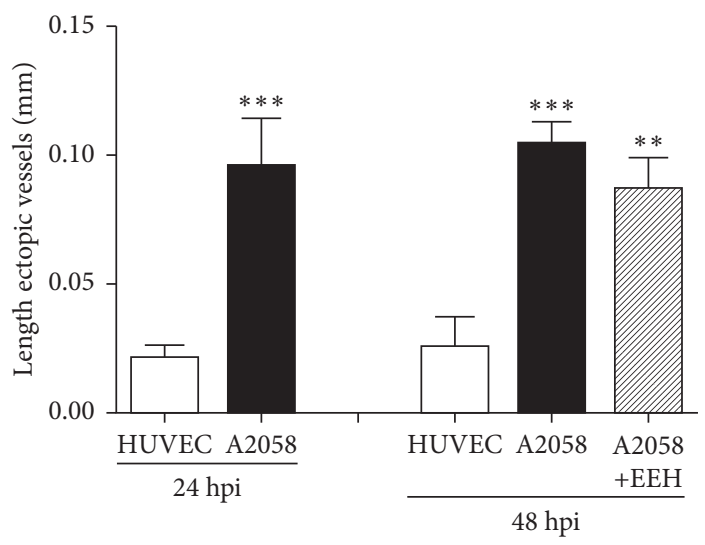

(h)

FIGURE 1: Euphorbia ebracteolata Hayata (EEH) inhibited SIV ectopic hyperplasia induced by melanoma cells in transgenic Tg (flk1:GFP) zebrafish embryos. Human melanoma cells were microinjected into zebrafish embryos at $48 \mathrm{hpf}$, and the resulting xenografts were imaged with a fluorescence microscope after 24 and $48 \mathrm{~h}$ (hpi), respectively. (a, d) SIV ectopic vessels in green fluorescence (red arrows) at 24 hpi zebrafish embryo (control). (b, e) SIV ectopic vessels (red arrows) at 48 hpi in zebrafish embryo (control). (c, f) SIV ectopic vessels after treatment with $50 \mu \mathrm{g} / \mathrm{mL}$ EEH for 48 (h). (g) The length of ectopic blood vessels in A2058 or HUVEC xenografts at 24 and 48 hpi and the effects after treatment with Euphorbia ebracteolata Hayata (EEH) $\left({ }^{*} p<0.05\right)$. (h) The number of ectopic blood vessels in A2058 or HUVEC xenografts at $24 \mathrm{hpi}$ and $48 \mathrm{hpi}$ and the effects after treatment with EEH $\left({ }^{*} p<0.05\right)$. Scale bar $=200 \mu \mathrm{m}$.

Injection of melanoma cells into zebrafish embryos not only resulted in the metastatic spread of melanoma cells but also induced angiogenesis (SIV) in zebrafish/tumor xenografts (Figure 1). Together, these results suggested that transplanted melanoma cells induced neovascularization in zebrafish/tumor xenografts. First, we found that EEH significantly inhibited the increase in length and number of ectopic blood vessels of the SIV. Next, we confirmed that $\mathrm{ECB}$ was the active ingredient of EEH and was more potent in inhibiting the length and number of ectopic blood vessels 


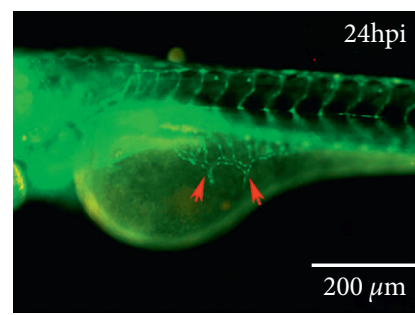

(a)

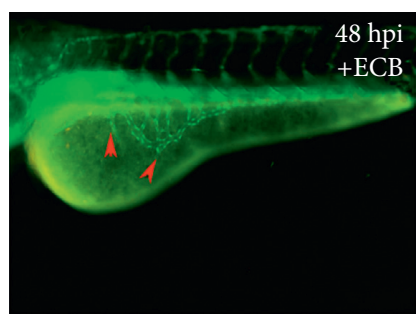

(c)

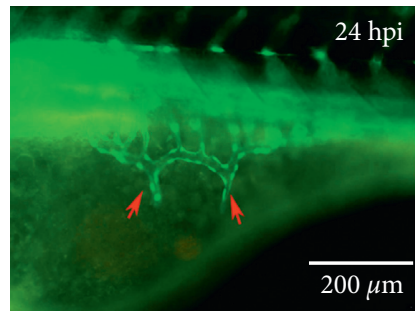

(e)

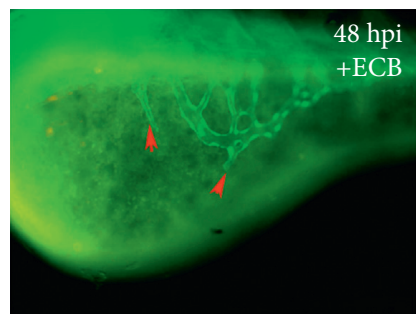

(g)

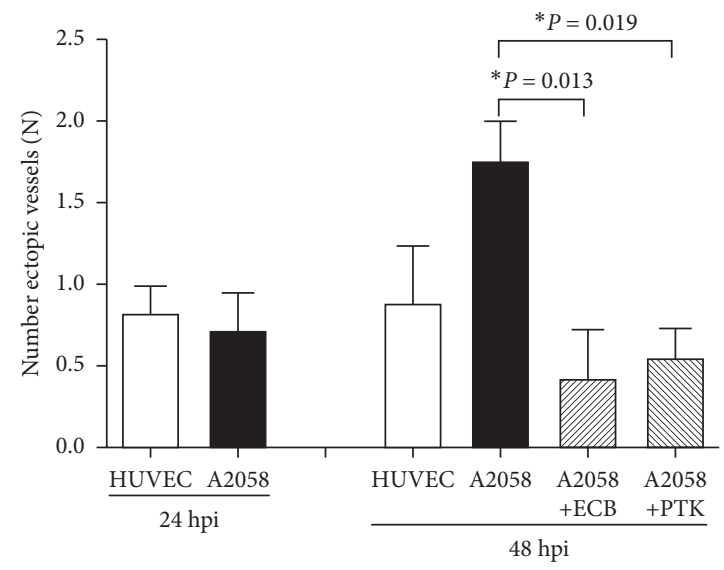

(i)

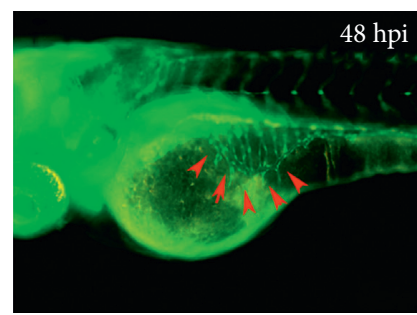

(b)

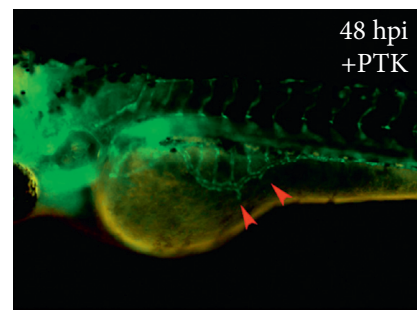

(d)

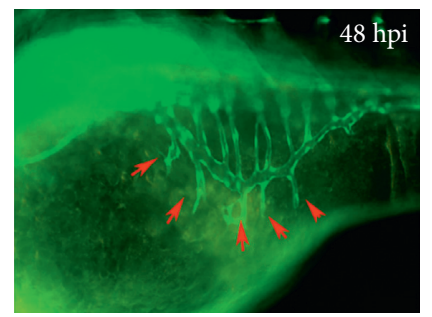

(f)

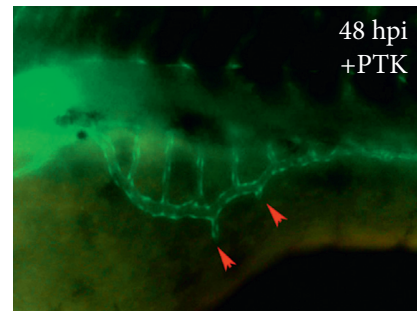

(h)

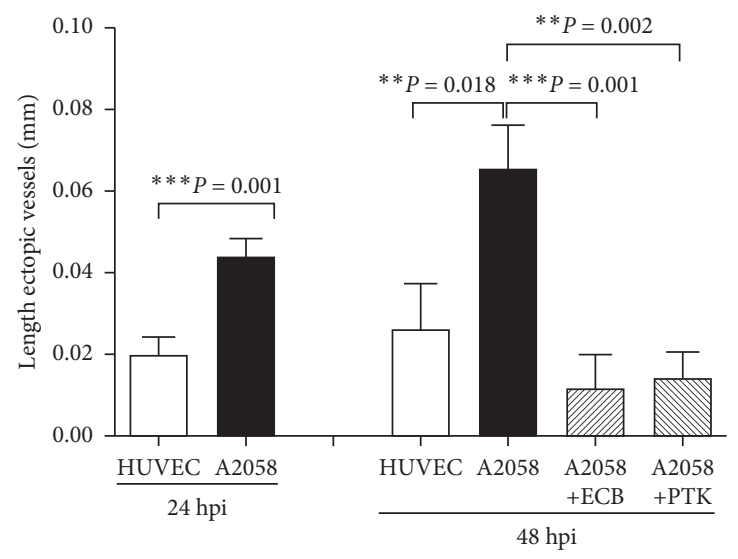

(j)

FIgURE 2: Euphorbiaceae compound B (ECB) inhibited SIV ectopic hyperplasia induced by melanoma cells in transgenic Tg (flk1:GFP) zebrafish embryos. Human melanoma cells were microinjected into zebrafish embryos at $48 \mathrm{hpf}$, and the resulting xenografts were imaged with a fluorescence microscope after 24 and $48 \mathrm{~h}$ (hpi), respectively. (a, e) SIV ectopic vessels in green fluorescence (red arrows) at 24 hpi zebrafish embryo (control). (b, f) SIV ectopic vessels (red arrows) at 48 hpi zebrafish embryo (control). (c, g) SIV ectopic vessels after treatment with $20 \mu \mathrm{g} / \mathrm{mL}$ ECB for $48 \mathrm{~h}$. (d, h) SIV ectopic vessels after treatment with $1 \mu \mathrm{M}$ PTK for $48 \mathrm{~h}$. (i) The length of ectopic blood vessels in A2058 or HUVEC xenografts at 24 and $48 \mathrm{hpi}$ and the effects after treatment with ECB and PTK $\left({ }^{*} p<0.05\right)$. (j) The number of ectopic blood vessels in A2058 or HUVEC xenografts at 24 hpi and 48 hpi and the effects after treatment with ECB or PTK $\left({ }^{*} p<0.05\right)$. Scale bar $=200 \mu \mathrm{m}$. 


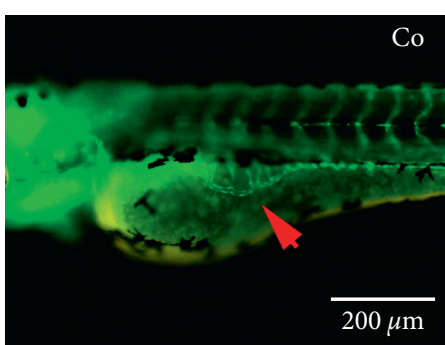

(a)

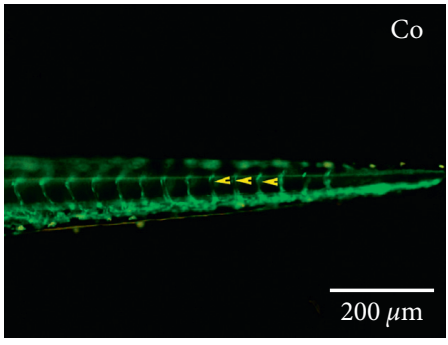

(d)

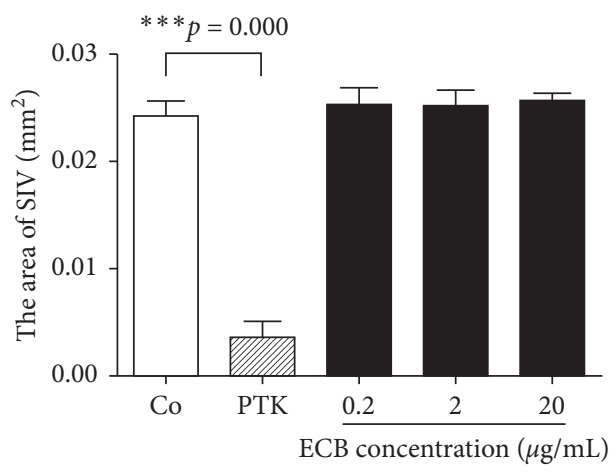

(g)

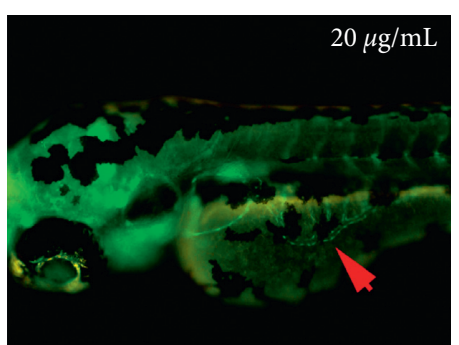

(b)

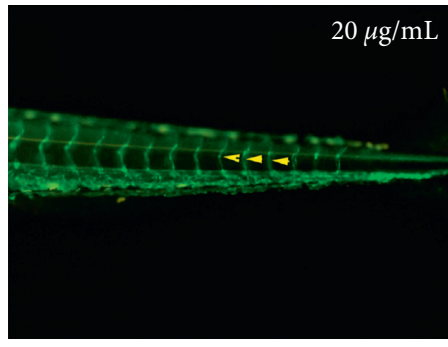

(e)

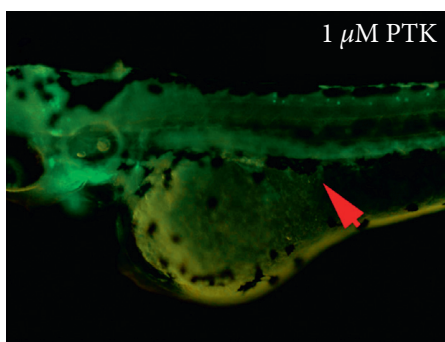

(c)

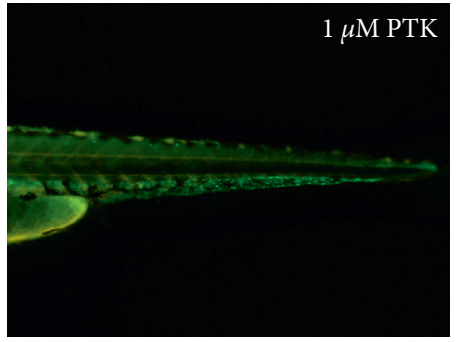

(f)

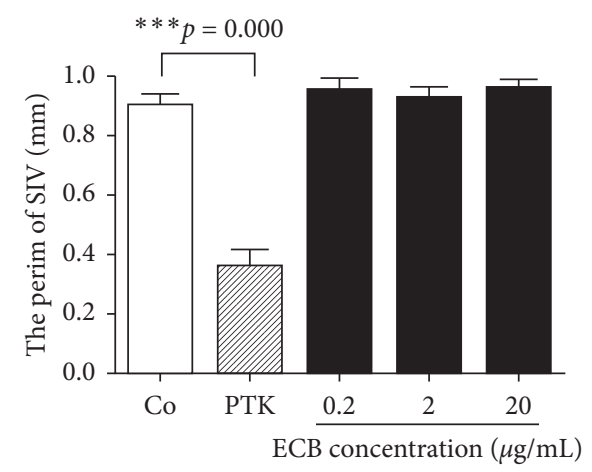

(h)

FIGURE 3: Effect of ECB on SIV and ISV angiogenesis in zebrafish embryos. Zebrafish embryos were imaged after treatment with ECB or PTK from 4 hpf to 72 hpf. (a, b) Control group. (b, e) ECB group. (c, f) PTK group. Red arrows point to SIV vessels above the yolk region $(\mathrm{a}-\mathrm{c})$. Yellow arrows show ISVs in the tail region $(\mathrm{d}-\mathrm{f})$. (g) The area covered by SIV vessels in zebrafish embryos $\left({ }^{* * *} p<0.001\right)$. (h) The perimeter (length) of SIV vessels in zebrafish embryos $\left({ }^{* * *} p<0.001\right)$. Scale bar $=200 \mu \mathrm{m}$.

of the SIV and inhibited the migration of A2058 cells in zebrafish/melanoma xenografts. Furthermore, ECB reduced the mRNA expression of zebrafish vegfa, vegfr 2 , and vegfr 3 and induced the mRNA expression of zebrafish p53 and casp $3 a$ in xenografts.

Remodeling of the vascular network was required to support the tissues needed for oxygen and nutrients during embryonic development [13]. The occurrence and metastasis of tumors increased the proliferation of blood vessels. Therefore, many anticancer drugs suppressed cancer by inhibiting angiogenesis [14]. Even for short-term treatment $(1 \mathrm{~h})$, the angiogenesis inhibitor SU5416 prevented new angiogenesis and angiogenic blood vessel formation. However, TNP470 required continuous exposure to block formation of the SIV and had no obvious effect on angiogenesis [15]. To screen chemical compounds, Manfred et al. made mitf::xmrk transgenic medaka. This was a stable transgenic melanoma model in which tumor development was observed in all gene carriers [16]. Here, we used a zebrafish/melanoma xenograft model to evaluate tumor- induced angiogenesis of the SIV in zebrafish embryos and showed that EEH or ECB inhibited the length of SIV ectopic blood vessels and reduced the number of blood vessels. Lenard et al. believed that the decrease in blood vessels was caused by cell self-fusion [13]. In addition, Koenig et al. suggested that Vegfa signaling can guide endothelial cells to migrate out of the existing vasculature and merge to form intestinal blood vessels. A similar mechanism may be used during angiogenesis in other organs [7]. Zhou et al. used the VEGFR1 antagonistic peptide F56 as an inhibitor to act on blood vessels and found that F56 did not affect VEGF-Ainduced endothelial cell proliferation but did reduce endothelial cell migration and angiogenesis. In addition, F56 inhibited angiogenesis of the chorioallantoic membrane in chicken embryos and the SIV in zebrafish embryo. Monomeric peptide F56 has significant antitumor activity by inhibiting angiogenesis [17]. Ponatinib is a vascular inhibitor that is used for FDA-approved cancer treatment [18] and can also inhibit the formation of ISVs and the SIV in zebrafish embryos. The antiangiogenic effect of 


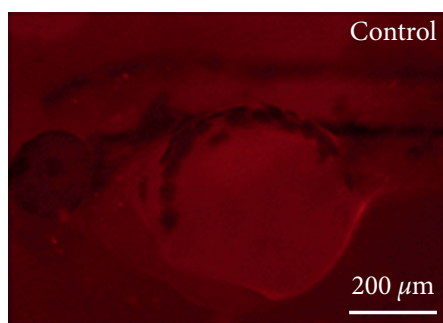

(a)

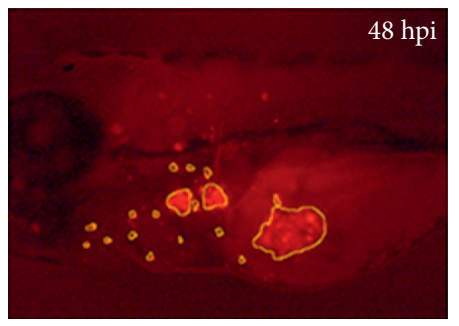

(d)

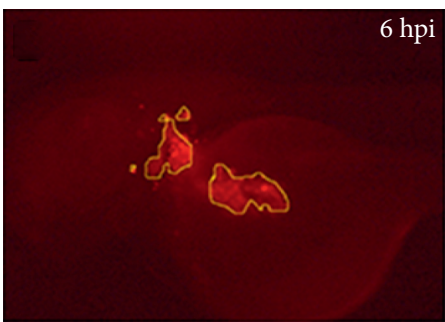

(b)

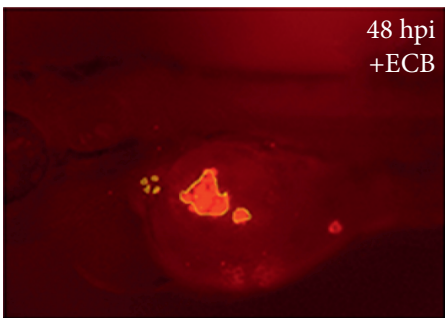

(e)

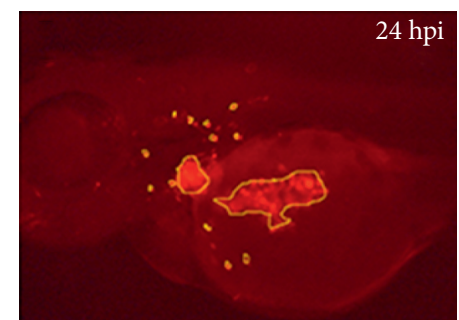

(c)

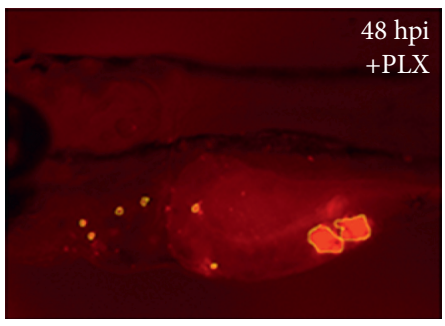

(f)

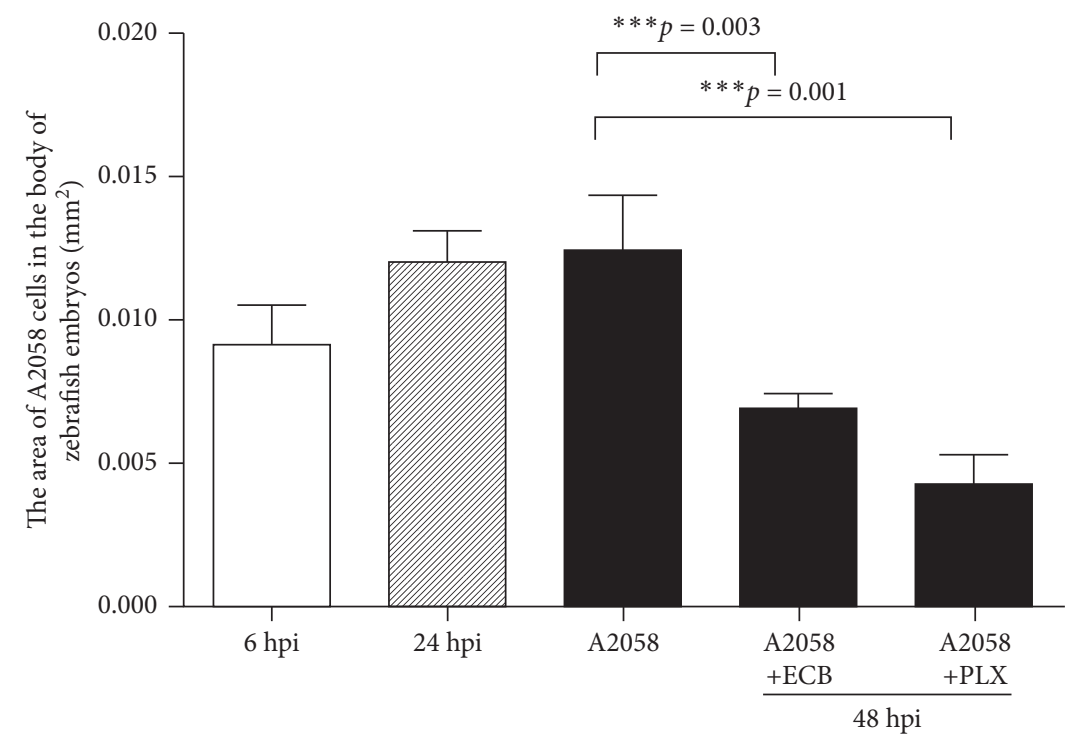

(g)

FIGURE 4: Effect of Euphorbiaceae compound B (ECB) on metastasis of melanoma cells in zebrafish xenografts. Zebrafish embryos were injected with A2058 cells at $48 \mathrm{hpf}$ and observed at $6 \mathrm{hpi}, 24 \mathrm{hpi}$, and $48 \mathrm{hpi}$, respectively. (a) Not injected. (b-d) A2058 cells were injected into zebrafish embryos at $48 \mathrm{hpf}$ and observed at $6 \mathrm{hpi}, 24 \mathrm{hpi}$, and $48 \mathrm{hpi}$, respectively. (e) A2058 cells were injected into zebrafish embryos and treated with $20 \mu \mathrm{g} / \mathrm{mL}$ ECB for $24 \mathrm{~h}$ and observed at $48 \mathrm{hpi}$. (f) A2058 cells were injected into zebrafish embryos and treated with $1 \mu \mathrm{M}$ PLX4720 (PLX) for $24 \mathrm{~h}$ and observed at $48 \mathrm{hpi}$. (g) The area of CM-DiI labeling (melanoma cell) in the zebrafish embryos. Scale bar $=200 \mu \mathrm{m} .{ }^{* * *}$ indicates a significant difference $(p<0.001)$.

Ponatinib on HUVECs was evaluated using cell proliferation and migration, angiogenesis, and wound-healing assays. Ponatinib inhibited Vegf-induced phosphorylation of Vegfr2 and its downstream signaling, including the Akt/ eNOS/NO pathway and the MAPK pathway (ERK and p38MAPK) [12]. In addition, 12-deoxyphorbol 13-palmitate in Euphorbia fischeriana Steud inhibited the Vegfr-2 signaling pathway, reduced microvessel density, inhibited VEGF, and blocked the PI3K/Akt/mTOR signaling pathway, thereby resulting in inhibition of MCF-7 breast cancer cell proliferation in mice $[19,20]$. Similar to these findings, we found that ECB significantly inhibited the ectopic vascular length of the SIV in zebrafish and inhibited the mRNA expression of vegf, vegfr2, and vegfr 3 in zebrafish xenografts.

Jolkinolide B isolated from Euphorbia fischeriana Steud induced apoptosis in a B16F10 mouse melanoma model by altering glycolysis. In addition, jolkinolide B treatment increased mRNA expression of the apoptosis gene Bax, Casp3, and Casp9, reduced mRNA expression of antiapoptosis genes $B c l 2$, reduced the mitochondrial membrane potential of B16F10 cells, and increased the level of reactive oxygen species (ROS), thereby having antitumor effects [21]. Moreover, 12-deoxyphorbol 13-palmitate isolated from 


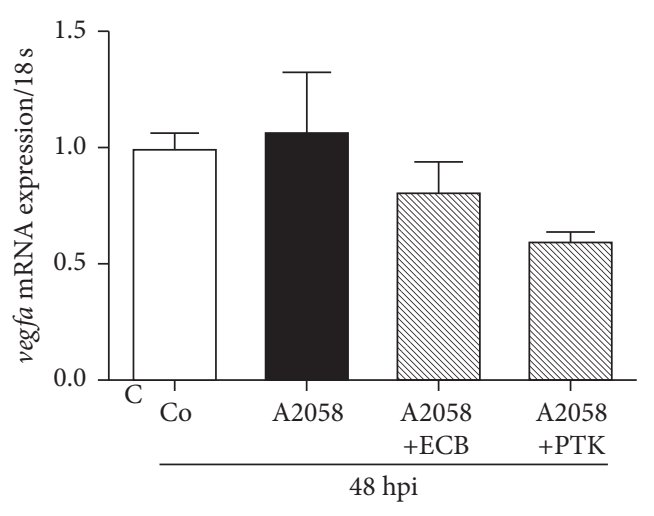

(a)

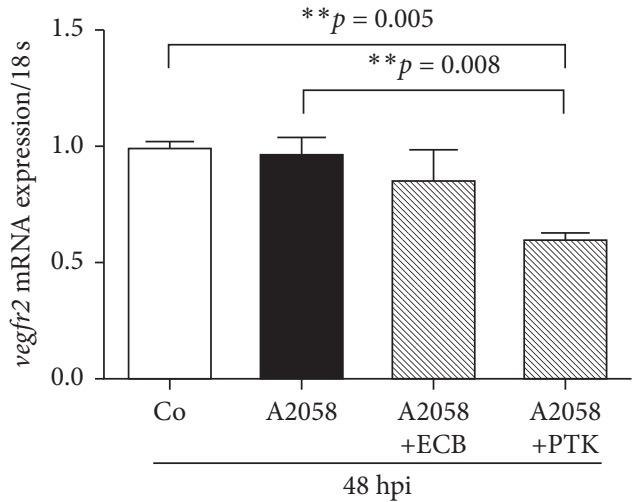

(b)

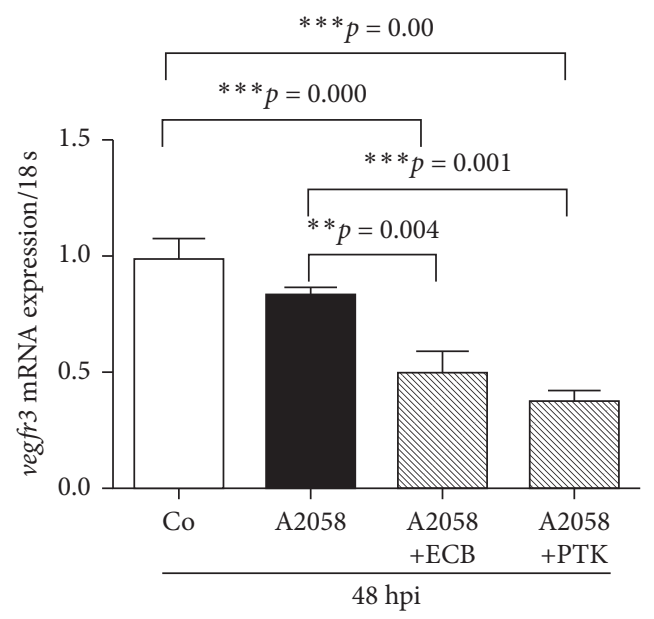

(c)

FIGURE 5: Effect of Euphorbiaceae compound B (ECB) on zebrafish vegfa, vegfr2, and vegfr 3 mRNA expression in xenografts using real-time PCR: (a) vegfa mRNA expression, (b) vegfr2 mRNA expression, and (c) vegfr 3 mRNA expression. ${ }^{* *}$ and ${ }^{* * *}$ indicate a significant difference $\left({ }^{* *} p<0.01 ;{ }^{* * *} p<0.001\right)$.

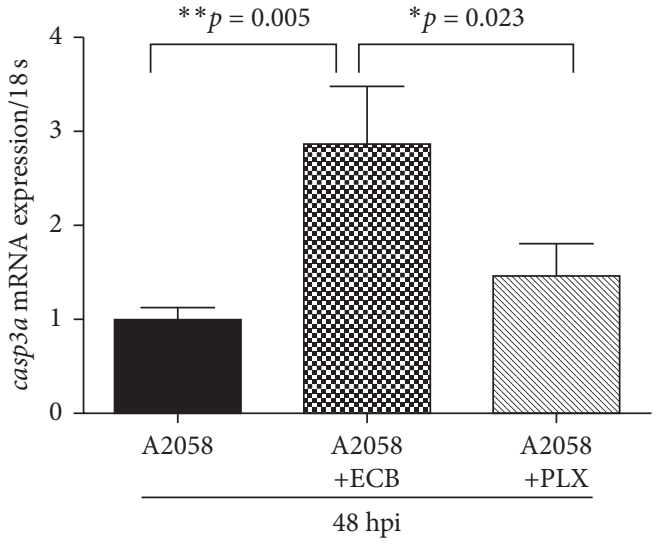

(a)

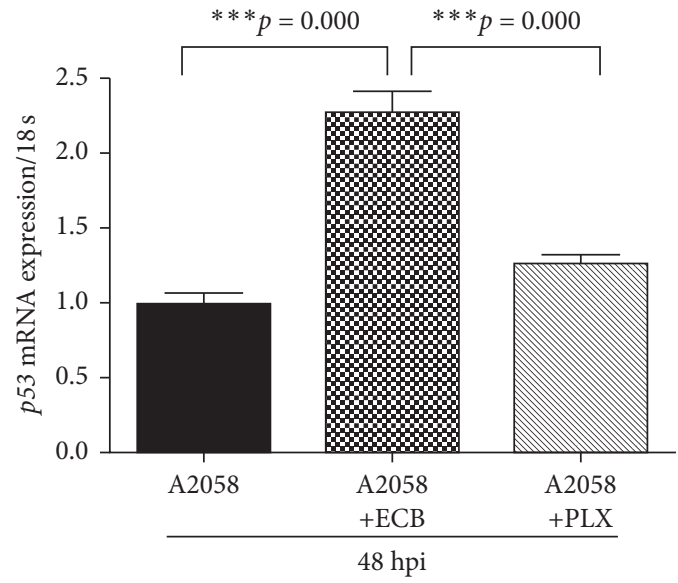

(b)

Figure 6: Euphorbiaceae compound B (ECB) affected the expression of zebrafish casp $3 a$ and $p 53 \mathrm{mRNA}$ in xenografts using real-time PCR. A2058 cells were labeled with CM-DiI, injected into zebrafish embryos at $48 \mathrm{hpf}$, and treated with $20 \mu \mathrm{g} / \mathrm{mL}$ ECB and $1 \mu \mathrm{M}$ PLX or the medium only control at $72 \mathrm{hpf}(24 \mathrm{hpi})$, respectively. Casp3a (a) and $p 53$ (b) mRNA expressions were quantified. ${ }^{*} p<0.05,{ }^{* *} p<0.01$, and ${ }^{* * *} p<0.001$. 
Euphorbia fischeriana Steud induced cell cycle arrest at the G2-M checkpoint of BGC823 cells and upregulated the expression of $p 53, p 21$, and $I \kappa B-\alpha$ in tumor cells, leading to tumor cell apoptosis and the inhibition of tumor growth [22]. In this study, a zebrafish/melanoma xenograft model was established to demonstrate that ECB from Euphorbia ebracteolata treatment also inhibited proliferative blood vessels, which was associated with increased mRNA expression of casp $3 a$ and $p 53$ in zebrafish.

In this study, we investigated the inhibitory effect of EEH or ECB on hyperplasia of blood vessels caused by human melanoma cells in zebrafish embryos. EEH and ECB inhibited melanoma-induced vascular proliferation of the SIV in zebrafish. This inhibitory effect may be achieved by blocking zebrafish vegfa and mRNA expression of the Vegf receptors vegfr 2 and vegfr 3 that regulate blood vessels. The decrease in blood vessels may be affected by the apoptotic pathway. It is noteworthy that ECB treatment only inhibited abnormal blood vessel development induced by melanoma, which is different from vascular inhibitors that universally inhibit blood vessel growth, including normal angiogenesis. Taken together, these data show that ECB may have potential therapeutic value for anticancer treatment.

\section{Data Availability}

The study data are available from the corresponding author upon request.

\section{Conflicts of Interest}

The authors declare no conflicts of interest.

\section{Acknowledgments}

This study was funded by the National Natural Science Foundation of China (81360508 and 82060672) to Jingfeng Yang; Inner Mongolia University for Nationalities, China (MDXK008), to $\mathrm{Wu}$ Dong; Inner Mongolia Grassland Talent Project, China (2020), to Wu Dong; Natural Science Foundation of Inner Mongolia Autonomous Region of China (2018MS08033 to Jingfeng Yang, 2020 MS08103 to Wu Dong); Open Project Program of Inner Mongolia Key Laboratory of Toxicant Monitoring and Toxicology, China (MDK2018027), to Wenjing Dong; and Open Project Program of Inner Mongolia Research Institute of Traditional Mongolian Medicine Engineering Technology, China (MDK2019051 and MDK2020002), to $\mathrm{Wu}$ Dong. TL is partially supported by grants U54MD012392 from NIH/NIMHD and U54CA156735 from NIH/NCI.

\section{Supplementary Materials}

Graphic abstract: Scheme for EEH or ECB inhibition of SIV ectopic hyperplasia induced by melanoma cells in transgenic $\operatorname{Tg}(f l k 1: G F P)$ zebrafish/tumor xenograft embryos. Table S1. Primer for quantitative real-time PCR. Figure S1. HPLC chromatogram of EEH. A: EEH extracts, peak 8: ECB, 2,4- dihydroxy-6-methylbenzoidone, and B: Reference substance (EBC) (Bao, Y, 2014). (Supplementary Materials)

\section{References}

[1] S. Singh, A. Zafar, S. Khan, and I. Naseem, "Towards therapeutic advances in melanoma management: an overview," Life Sciences, vol. 174, pp. 50-58, 2017.

[2] Y. Ma, H. Wang, and M. Guo, "Stainless steel wire mesh supported molecularly imprinted composite membranes for selective separation of ebracteolata compound B from Euphorbia fischeriana," Molecules, vol. 24, p. 3, 2019.

[3] A. Wang, X. Gao, X. Huo et al., "Antioxidant acetophenone glycosides from the roots of Euphorbia ebracteolata Hayata," Natural Product Research, vol. 32, no. 18, pp. 2187-2192, 2018.

[4] R. Zhou, J. M. Curry, L. D. Roy et al., "A novel association of neuropilin-1 and MUC1 in pancreatic ductal adenocarcinoma: role in induction of VEGF signaling and angiogenesis," Oncogene, vol. 35, no. 43, pp. 5608-5618, 2016.

[5] K. S. Moshal, K. F. Ferri-Lagneau, and T. Leung, "Zebrafish model: worth considering in defining tumor angiogenesis," Trends in Cardiovascular Medicine, vol. 20, no. 4, pp. 114-119, 2010.

[6] K. S. Moshal, K. F. Ferri-Lagneau, J. Haider, P. Pardhanani, and T. Leung, "Discriminating different cancer cells using a zebrafish in vivo assay," Cancers, vol. 3, no. 4, pp. 4102-4113, 2011.

[7] A. L. Koenig, K. Baltrunaite, N. I. Bower et al., "Vegfa signaling promotes zebrafish intestinal vasculature development through endothelial cell migration from the posterior cardinal vein," Developmental Biology, vol. 411, no. 1, pp. 115-127, 2016.

[8] J. Drevs, R. Müller-Driver, C Wittig et al., "PTK787/ZK 222584 , a specific vascular endothelial growth factor-receptor tyrosine kinase inhibitor, affects the anatomy of the tumor vascular bed and the functional vascular properties as detected by dynamic enhanced magnetic resonance imaging," Cancer Research, vol. 62, no. 14, pp. 4015-4022, 2002.

[9] A. K. A. Gaumann, H. C. A. Drexler, S. A. Lang et al., "The inhibition of tyrosine kinase receptor signalling in leiomyosarcoma cells using the small molecule kinase inhibitor PTK787/ZK222584 (Vatalanib)," International Journal of Oncology, vol. 45, no. 6, pp. 2267-2277, 2014.

[10] C. Liu, W. Peng, C. Xu et al., "BRAF inhibition increases tumor infiltration by $\mathrm{T}$ cells and enhances the antitumor activity of adoptive immunotherapy in mice," Clinical Cancer Research, vol. 19, no. 2, pp. 393-403, 2013.

[11] Y. Bao, Analysis of HPLC Chromatographic Fingerprint of Mongolian Medicine Euphorbia Fischeriana Steud and HPLC-MSn Analysis of its Different Processing Products, Inner Mongolia University for Nationalities, Tongliao, China, 2014.

[12] N. Ai, C.-M. Chong, W. Chen et al., "Ponatinib exerts antiangiogenic effects in the zebrafish and human umbilical vein endothelial cells via blocking VEGFR signaling pathway," Oncotarget, vol. 9, no. 62, pp. 31958-31970, 2018.

[13] A. Lenard, S. Daetwyler, C. Betz, E. Ellertsdottir, H. G. Belting, and J. Huisken, "Endothelial cell self-fusion during vascular pruning," PLoS Biology, vol. 13, no. 4, Article ID e1002126, 2015.

[14] H. Luo, C. T. Vong, H. Chen, Y. Gao, P. Lyu, and L. Qiu, "Naturally occurring anti-cancer compounds: shining from Chinese herbal medicine," Chinese Medicine, vol. 14, p. 48, 2019. 
[15] G. N. Serbedzija, E. Flynn, and C. E. Willett, "Zebrafish angiogenesis: a new model for drug screening," Angiogenesis, vol. 3, no. 4, pp. 353-359, 1999.

[16] S. Manfred, W. Brigitta, A. G. C. L. Juliette, T. Yoshihito, T. Shunichi, and M. Svenja, "A mutated EGFR is sufficient to induce malignant melanoma with genetic background-dependent histopathologies," Journal of Investigative Dermatology, vol. 130, no. 1, pp. 249-258, 2010.

[17] Z. Zhou, C. Zhao, L Wang et al., "A VEGFR1 antagonistic peptide inhibits tumor growth and metastasis through VEGFR1-PI3K-AKT signaling pathway inhibition," American Journal of Cancer Research, vol. 5, no. 10, pp. 3149-3161, 2015.

[18] J. Kim, A. Nair, P. Keegan et al., "Evaluation of serious postmarket safety signals within 2 Years of FDA approval for new cancer drugs," The Oncologist, vol. 25, no. 4, pp. 348-354, 2019.

[19] H.-Y. Xu, Y.-M. Pan, Z.-W. Chen et al., "12-Deoxyphorbol 13palmitate inhibit VEGF-induced angiogenesis via suppression of VEGFR-2-signaling pathway," Journal of Ethnopharmacology, vol. 146, no. 3, pp. 724-733, 2013.

[20] Y. Yang, H. Cong, C. Han, L. Yue, H. Dong, and J. Liu, “12Deoxyphorbol 13-palmitate inhibits the expression of VEGF and HIF- $1 \alpha$ in MCF-7 cells by blocking the PI3K/Akt/mTOR signaling pathway," Oncology Reports, vol. 34, no. 4, pp. 1755-1760, 2015.

[21] C. Gao, X. Yan, B. Wang, L. Yu, J. Han, and D. Li, "Jolkinolide $\mathrm{B}$ induces apoptosis and inhibits tumor growth in mouse melanoma B16F10 cells by altering glycolysis," Science Reports, vol. 6, p. 36114, 2016.

[22] H.-Y. Xu, Z.-W. Chen, H. Li et al., "12-Deoxyphorbol 13palmitate mediated cell growth inhibition, G2-M cell cycle arrest and apoptosis in BGC823 cells," European Journal of Pharmacology, vol. 700, no. 1-3, pp. 13-22, 2013. 